HIV-1 envelope glycoprotein, no vaccination strategy has successfully produced such antibodies. Thus, the ability to optimise and deliver HIV envelope immunogens that can induce bnAb has remained a formidable challenge.

Methods To optimise immunogens for inducing bnAb, our group has pursued two main directions. In the first instance we developed B cell immunogens mimicking the native HIV-1 enveloped gp120 glycoprotein. Supernatants of stably transfected mutant lec1 $\mathrm{CHO}$ cells using a flag tag. We next assess antibodies isotypes specific to this immunogen in plasma obtained from antiretroviral naive participants. Secondly, through surface engineering of the evolutionary phage Qbeta we built in several epitopes of bnAbs for effective delivery to the immune system. We next assess in plasma from 648 seropositive participants the abundance of antibody isotypes specific to these B cell immunogens.

Results The results obtained showed that all IgG isotypes were detected for both the manosylated and $\mathrm{CHO}$ wild-type expressed gp120. Although all IgG antibody isotypes including $\operatorname{IgG} 1, \operatorname{IgG} 2, \operatorname{IgG} 3$ and IgG4 were dictated, there was no significant difference between antibody titres directed to manosylated gp120 and wild-type gp120. Well over $87 \%$ of seropositive participants showed specific antibody responses to conserved B cell epitopes displayed on the surface of Qbeta phage.

Conclusions These novel immunogens can be used as vaccine candidates.

\title{
OA-030 IMMUNOGENS DESIGNED FOR TARGETING NEUTRALIZING EPITOPES OF HIV-1 ENVELOPE GLYCOPROTEIN
}

Godwin Nchinda, ${ }^{1}$ Alain Bopda, ${ }^{2}$ Nadesh Nji, ${ }^{1}$ Georgia Ambada, ${ }^{1}$ Loveline Ngu, ${ }^{1}$ Jules Tchadji, ${ }^{3}$ Carol Sake, ${ }^{3}$ Suzanne Magagoum, ${ }^{3}$ Ghislain D. Njambe, ${ }^{3}$

Abel Lisom, ${ }^{3}$ Chae Gyu Park ${ }^{4}$ ' $C I R C B$, Cameroon; ${ }^{2}$ Alabama State University, United States of America; ${ }^{3}$ University of Yaoundé I, Cameroon; ${ }^{4}$ YUCM (SBSI), Korea

\subsection{6/bmjgh-2016-000260.36}

Background Due to its unique challenge of establishing lifelong reservoirs a successful HIV-1 vaccine must elicit protective antibodies responses at the portals of entry. Broadly neutralising antibodies (bnAb) have been demonstrated not only to be therapeutic through suppressing viraemia in HIV-1 infected people but also preventive in blocking HIV-1 infection in animal models. This implies that a desirable HIV-1 vaccine candidate should be able to induce HIV-1 specific bnAb with an extensive ability to neutralise a broad range of HIV-1 isolates. Although several bnAb are known to target conserved regions in the 